\title{
Theoretical Informatics and Applications
}

Theoret. Informatics Appl. 37 (2003) 273-299

DOI: $10.1051 /$ ita:2003020

\section{TRACED PREMONOIDAL CATEGORIES}

\author{
NiCK Benton ${ }^{1}$ AND MARTin Hyland ${ }^{2}$
}

\begin{abstract}
Motivated by some examples from functional programming, we propose a generalization of the notion of trace to symmetric premonoidal categories and of Conway operators to Freyd categories. We show that in a Freyd category, these notions are equivalent, generalizing a well-known theorem relating traces and Conway operators in Cartesian categories.
\end{abstract}

Mathematics Subject Classification. 68N18, 03B70, 03G30.

\section{INTRODUCTION}

Monads were introduced into computer science by Moggi [25] as a structuring device in denotational semantics and soon became a popular abstraction for writing actual programs, particularly for expressing and controlling side-effects in "pure" functional programming languages such as Haskell $[24,33]$. Power and Robinson subsequently introduced premonoidal categories as a generalization of Moggi's computational models [30], whilst Hughes developed arrows, which are the equivalent programming abstraction [18].

Some uses of monads in functional programming seem to call for a kind of recursion operator on computations for which, informally, the recursion "only takes place over the values". For example, the Haskell Prelude defines the (internally implemented) ST and IO monads for, respectively, potentially state-manipulating and input/output-performing computations. These come equipped with polymorphic functions

Keywords and phrases. Traces, fixed point operators, premonoidal categories, recursion, monads.

1 Microsoft Research, Roger Needham Building, 7 J J Thomson Avenue, Cambridge CB3 0FB, UK; e-mail: nick@microsoft.com

${ }^{2}$ University of Cambridge, Department of Pure Mathematics and Mathematical Statistics, Wilberforce Road, Cambridge CB3 0WB, UK; e-mail: M.Hyland@dpmms.cam.ac.uk 
fixST $::(a->$ ST a) $\rightarrow$ ST a
fixIO $::(a \rightarrow$ IO a) $\rightarrow$ IO a

which allow computations to be recursively defined in terms of the values they produce. For example, the following program uses fixIo ${ }^{1}$ to extend a cunning cyclic programming trick due to Bird [1] to the case of side-effecting computations. replacemin computes a tree in which every leaf of the argument has been replaced by the minimum of all the leaves. It does this in a single pass over the input and prints out each leaf as it encounters it ${ }^{2}$ :

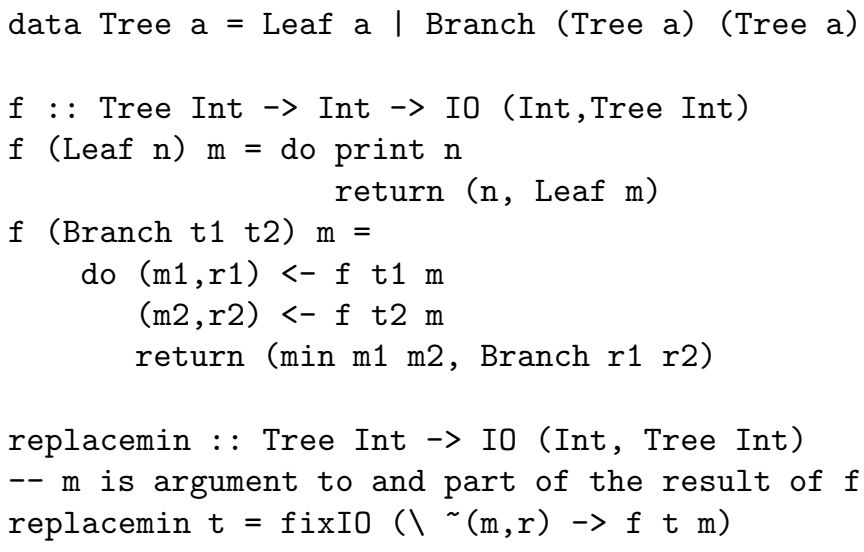

As another (though still somewhat contrived) example, consider modeling the heap of a fictitious pure Scheme-like language at a fairly low level. One might interpret heap-manipulating computations using a monad $\mathrm{T}$ that is an instance of a type class something like this

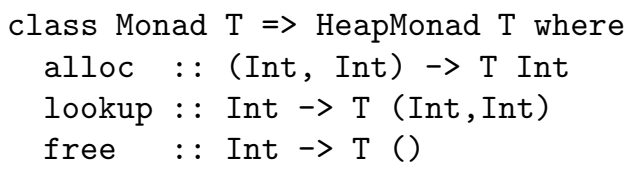

The intention is that alloc takes two integers and returns a computation which finds a free cons cell in the heap, fills it with those two integers and returns the (strictly positive) address of the allocated cell. lookup takes an integer address and returns the contents of that cons cell, whilst free marks a particular address as available for future allocations. Since the values in the $c a r$ and $c d r$ of cells can be used as the addresses of other cells, we can interpret programs which build data structures such as lists in the heap. What if the language we are interpreting can create cyclic structures (for example, closures for recursive functions)? At the machine level, cyclic structures are created by allocating cells containing dummy

\footnotetext{
${ }^{1}$ We should note that fixI0 does not actually satisfy the axioms we will propose. However, the basic pattern would remain the same, though the code would be a little longer, if we had performed side-effects involving state instead.

$2_{\text {The tilde }}$ on the last line specifies "lazy" pattern matching for the pair (m,r). Haskell's tuples are actually lifted products and pattern matching is, by default, strict. Without the tilde the function would diverge.
} 
values and then "tying the knot" by overwriting those dummy values with the addresses returned by the allocator. Hence we could just provide destructive update operations

setcar : : Int $\rightarrow$ Int $\rightarrow \mathrm{T}($ )

and use those to create cycles. However, if the interpreted language itself does not include destructive assignment, but only creates cycles using higher-level constructs, then adding assignment operations to the monad breaks an abstraction barrier. One solution is to add a recursion operation to the monad

$$
\text { fixT : : (a } \rightarrow \mathrm{T} \text { a) } \rightarrow \mathrm{T} \text { a }
$$

with a definition such that the following code creates a two-element cyclic list (and returns the addresses of both cells):

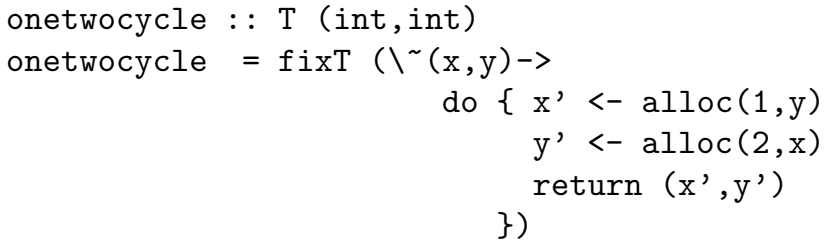

Observe that although the computation is recursively defined, it should only perform the two allocation side-effects once.

Many of the real uses of this kind of recursion have the flavour of the previous example: they involve computations which create cyclic structures for which the identity, order of creation or multiplicity of creation of the objects in the structure is significant. An interesting example arises in work on using Haskell to model hardware. Early versions of both Lava [2] and Hawk [23] specified circuits in a monadic style, instantiating the monad differently for different applications (such simulating the circuit, generating a netlist or interfacing with a theorem prover). Cyclic circuits (i.e. those with feedback) were defined in essentially the style used to define onetwocycle above. Lava has moved away from that style, in part because it is syntactically awkward ${ }^{3}$. Launchbury et al. [23] also noted that programming in a monadic style with $\mathrm{fixT}$ is uncomfortable, and suggested extending Haskell's do notation to allow recursive bindings. That suggestion was followed up by Launchbury and Erkök, who proposed an axiomatization of operators like fixt (which they call $\mathrm{mfix}$ ) and showed how the do notation can be extended to allow recursive bindings in the case that the underlying monad supports such an mfix operation $[9,22]$.

Launchbury and Erkök's axiomatization of mfix is partly in terms of equations and partly in terms of inequations, intended to be interpreted in the "usual" (slightly informal) concrete domain theoretic model of Haskell. One striking feature of [22] is that it does not appear to build on any of the large body of existing

\footnotetext{
${ }^{3}$ Lava now uses a modified version of Haskell with "observable sharing": allowing new name generation as an implicit side-effect of every expression and hence changing the equational theory of the language [6].
} 
work on axiomatic/categorical treatments of recursion, even those (such as [8]) which consider fixed points in terms of monads. The authors cite some of this work but state, quite correctly, that the non-standard kind of recursion in which they are interested is different from that covered in the literature. Although the presence of a fixpoint object [8], for example, allows an operator with the same type as mfix to be defined, it is not of the kind we want.

From a categorical perspective, we seem to want a notion of recursion or feedback on the Kleisli category of a CCC with a strong monad. There is a special case of this situation in which earlier work does provide an answer. Although none of Launchbury and Erkök's examples are of commutative monads, in that case the Kleisli category will be symmetric monoidal and Joyal, Street and Verity's notion of trace seems to fit the bill [21].

In the general case of a non-commutative monad, however, the Kleisli category will only be symmetric premonoidal. The work described here grew firstly from the natural mathematical question of what the right definition of traced premonoidal category might be, and secondly from wondering whether an answer might provide a sensible categorical semantics for the kind of fixpoint operators described in [22]. We give a natural, straightforward and well-behaved answer to the first question, though it only accounts for a rather special subset of the cases considered by Launchbury and Erkök.

\section{BACKGROUND}

\subsection{Premonoidal categories}

For a careful definition of the notion of (symmetric) premonoidal category and (symmetric) premonoidal functor, see Power and Robinson's paper [30]. Briefly, a premonoidal category is a monoidal category except that the tensor product $\otimes$ need only be a functor in each of the two variables separately. Thus if $f: A \rightarrow B$ and $g: A^{\prime} \rightarrow B^{\prime}$ in a premonoidal category $\mathbb{K}$ then the two evident morphisms $A \otimes A^{\prime} \rightarrow B \otimes B^{\prime}$

$$
\begin{aligned}
& f \ltimes g=A \otimes A^{\prime} \stackrel{f \otimes A^{\prime}}{\longrightarrow} B \otimes A^{\prime} \stackrel{B \otimes g}{\longrightarrow} B \otimes B^{\prime} \\
& f \rtimes g=A \otimes A^{\prime} \stackrel{A \otimes g}{\longrightarrow} A \otimes B^{\prime} \stackrel{f \otimes B^{\prime}}{\longrightarrow} B \otimes B^{\prime}
\end{aligned}
$$

are not generally equal.

We generally write $I$ for the unit of the tensor in a (pre)monoidal category, $\sigma$ for the symmetry if there is one, and $\lambda, \rho, \alpha$ for the natural isomorphisms

$$
\begin{aligned}
\lambda & : \quad I \otimes A \rightarrow A \\
\rho & : \quad A \otimes I \rightarrow A \\
\alpha & : \quad(A \otimes B) \otimes C \rightarrow A \otimes(B \otimes C) .
\end{aligned}
$$


However, since we have coherence theorems for (symmetric) (pre)monoidal categories [30], we will usually elide the structural isomorphisms.

Definition 2.1. A morphism $f: A \rightarrow B$ in a premonoidal category $\mathbb{K}$ is central if for all $g: A^{\prime} \rightarrow B^{\prime}$ in $\mathbb{K}, f \ltimes g=f \rtimes g$. If at least one of $f$ and $g$ is central, then we may unambiguously write $f \otimes g$. The centre $Z(\mathbb{K})$ of a premonoidal category $\mathbb{K}$ is the monoidal subcategory of $\mathbb{K}$ with the same objects but only the central morphisms.

The inclusion functor $Z(\mathbb{K}) \rightarrow \mathbb{K}$ is a strict, identity-on-objects premonoidal functor (and symmetric if $\mathbb{K}$ is). In more recent work Power in particular has stressed the importance from the algebraic point of view in having an explicit choice of centre. That is, one is interested in the situation where one has a functor $J: \mathbb{M} \rightarrow \mathbb{K}$ from a specified (symmetric) monoidal subcategory of a (symmetric) premonoidal $\mathbb{K} ; J$ factors through $Z(\mathbb{K})$, so this amounts to specifying a particular subcategory of central morphisms. (For many results $J$ does not even need to be faithful, but we do not consider that generality here.) We call a $J: \mathbb{M} \rightarrow \mathbb{K}$ as above a centered premonoidal category, but since this is our preferred notion we usually drop the "centered". In this context, by central morphisms we shall mean the morphisms of $\mathbb{M}$. One should think of $\mathbb{M}$ as a category of values and $\mathbb{K}$ as a category of possibly-effectful computations. An important special case is the following:

Definition 2.2. A Freyd category [31] is specified by a Cartesian category $\mathbb{C}$, a symmetric premonoidal category $\mathbb{K}$ and an identity-on-objects strict symmetric premonoidal functor $J: \mathbb{C} \rightarrow \mathbb{K}$.

Note that morphisms in the specified centre of a Freyd category are "pure" not merely in the sense of commuting with arbitrary effectful computations, but also in being copyable and discardable.

Example 2.3. If $T$ is a strong monad on a symmetric monoidal category $\mathbb{M}$, then the Kleisli category $\mathbb{M}_{T}$ is symmetric premonoidal and the canonical functor from $\mathbb{M}$ to $\mathbb{M}_{T}$ is strict symmetric premonoidal. Thus in the case that $\mathbb{M}$ is Cartesian, we have a Freyd category. If the monad is commutative, then $\mathbb{M}_{T}$ is symmetric monoidal and $J: \mathbb{M} \rightarrow \mathbb{M}_{T}$ is strict symmetric monoidal.

\subsection{TRACES AND FIXPOINTS}

The notion of traced monoidal category was introduced in [21]. The use of traces to interpret recursion in programming languages and the relationship between traces and fixpoints have attracted much attention in recent years, beginning with Hasegawa's thesis [16]. Categorical axiomatizations of fixpoint operators have been extensively studied, see $[4,8,27]$ for example; a particularly crisp and up-to-date account appears in [32]. 
Definition 2.4. A trace on a symmetric monoidal category $(\mathbb{M}, \otimes, I, \lambda, \rho, \alpha, \sigma)$ is a family of functions

$$
\operatorname{tr}_{A, B}^{U}: \mathbb{M}(A \otimes U, B \otimes U) \rightarrow \mathbb{M}(A, B)
$$

satisfying the following conditions:

Naturality in $A$ (Left Tightening).

If $f: A^{\prime} \otimes U \rightarrow B \otimes U, g: A \rightarrow A^{\prime}$ then

$$
\operatorname{tr}_{A, B}^{U}((g \otimes U) ; f)=g ; \operatorname{tr}_{A^{\prime}, B}^{U}(f): A \rightarrow B .
$$

Naturality in $B$ (Right Tightening).

If $f: A \otimes U \rightarrow B^{\prime} \otimes U, g: B^{\prime} \rightarrow B$ then

$$
\operatorname{tr}_{A, B}^{U}(f ;(g \otimes U))=\operatorname{tr}_{A, B^{\prime}}^{U}(f) ; g: A \rightarrow B .
$$

Dinaturality (Sliding). If $f: A \otimes U \rightarrow B \otimes V, g: V \rightarrow U$ then

$$
\operatorname{tr}_{A, B}^{U}(f ;(B \otimes g))=\operatorname{tr}_{A, B}^{V}((A \otimes g) ; f): A \rightarrow B .
$$

Action (Vanishing). If $f: A \rightarrow B$ then

$$
\operatorname{tr}_{A, B}^{I}\left(\rho ; f ; \rho^{-1}\right)=f: A \rightarrow B
$$

and if $f: A \otimes(U \otimes V) \rightarrow B \otimes(U \otimes V)$ then

$$
\operatorname{tr}_{A, B}^{U \otimes V}(f)=\operatorname{tr}_{A, B}^{U}\left(\operatorname{tr}_{A \otimes U, B \otimes U}^{V}\left(\alpha ; f ; \alpha^{-1}\right)\right) .
$$

Superposing. If $f: A \otimes U \rightarrow B \otimes U$ then

$$
\begin{aligned}
& \operatorname{tr}_{C \otimes A, C \otimes B}^{U}\left(\alpha ; C \otimes f ; \alpha^{-1}\right) \\
& \quad=C \otimes \operatorname{tr}_{A, B}^{U}(f): C \otimes A \rightarrow C \otimes B .
\end{aligned}
$$

Yanking. For all $U, \operatorname{tr}_{U, U}^{U}\left(\sigma_{U, U}\right)=U: U \rightarrow U$.

Monoidal categories provide a formal basis for reasoning about many of the graphical "boxes and wires" notations used in computer science. A precise foundation for this has been sketched by Hyland and Power [19]. Traced monoidal categories provide a formal basis for circuit-like notations involving feedback or cycles. In one reading at least, they are the building blocks for Girard's Geometry of Interaction [15]. We recall a result of Joyal, Street and Verity [21]:

Theorem 2.5. The forgetful 2-functor from the 2-category of compact closed categories to that of traced symmetric monoidal categories has a left biadjoint.

The basic Geometry of Interaction construction is an explicit form of this biadjoint 2-functor.

We present the trace axioms graphically in Figure 1, though we do not consider the formal semantics of such diagrams here. 


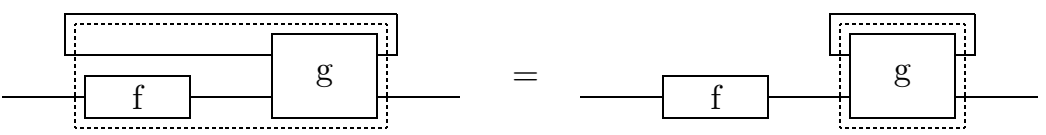

Left Tightening

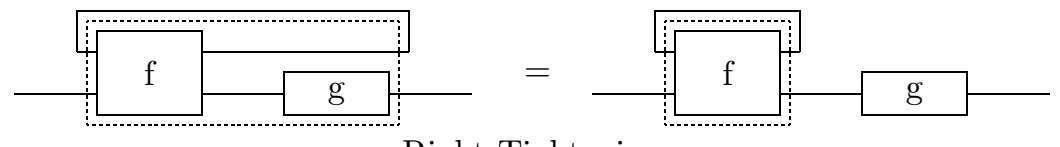

Right Tightening
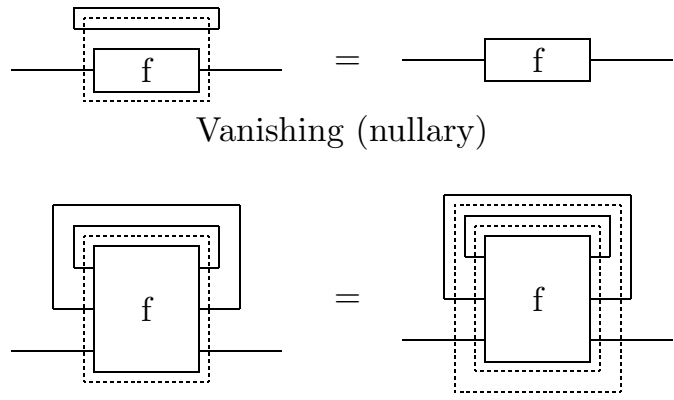

Vanishing (binary)
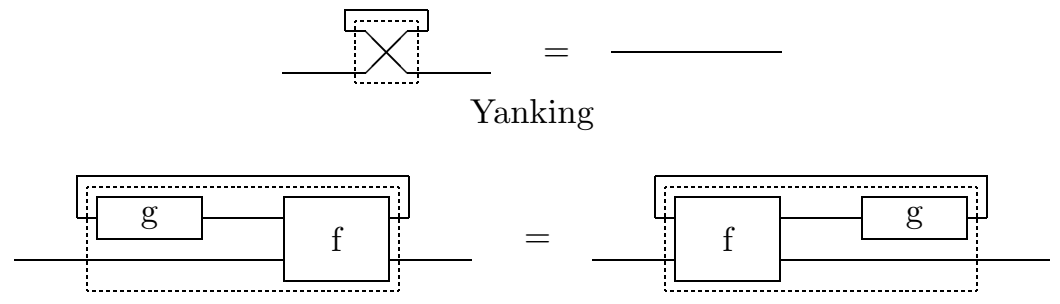

Sliding

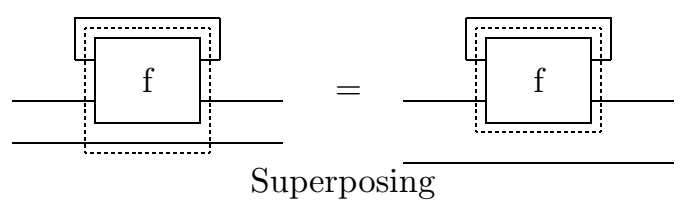

Figure 1. Trace axioms.

Definition 2.6. A parameterized fixpoint operator on a Cartesian category $\mathbb{C}$ is a family of functions

$$
(\cdot)^{\dagger}: \mathbb{C}(A \times U, U) \rightarrow \mathbb{C}(A, U)
$$

satisfying 
Naturality. If $f: B \times U \rightarrow U$ and $g: A \rightarrow B$ then

$$
g ; f^{\dagger}=((g \times U) ; f)^{\dagger}: A \rightarrow U .
$$

Fixed Point Property. If $f: A \times U \rightarrow U$ then

$$
\left\langle A, f^{\dagger}\right\rangle ; f=f^{\dagger}: A \rightarrow U .
$$

The above definition is rather weak. Well-behaved fixpoint operators typically satisfy other interesting conditions.

Definition 2.7. A Conway operator is a parameterized fixpoint operator which additionally satisfies

Dinaturality. If $f: A \times U \rightarrow V$ and $g: V \rightarrow U$ then

$$
(f ; g)^{\dagger}=(A \times g ; f)^{\dagger} ; g: A \rightarrow U .
$$

Diagonal Property. If $f: A \times U \times U \rightarrow U$ then

$$
((A \times \Delta) ; f)^{\dagger}=\left(f^{\dagger}\right)^{\dagger}: A \rightarrow U .
$$

The Conway operator axioms are shown graphically in Figure 2. Our terminology follows that of Bloom and Ésik [4], though they consider the opposite situation. They work with categories with co-products "generated by a single object", that is, they consider the opposite of Lawvere theories. For them a Conway theory is the opposite of a Lawvere theory equipped with a Conway operator in our sense. Readers familiar with [4] will observe the following:

- Our naturality corresponds to their parameter identity;

- Our fixed point property corresponds to their fixed point equation;

- Our dinaturality corresponds to their simplified composition identity;

- Our diagonal property corresponds to their double dagger identity.

In all cases, the extension from Lawvere theories to general Cartesian categories is routine. To the extent that our terminology differs from that of [4], it draws attention to familiar category-theoretic notions.

We make a few remarks on axiomatization. The axioms for a Conway operator imply various other useful properties including the Bekic property (allowing simultaneous fixed points to be reduced to sequential ones) and parameterized dinaturality (corresponding to the composition identity of [4]):

Definition 2.8. A fixpoint operator on a Cartesian category satisfies parameterized dinaturality if whenever $f: A \times U \rightarrow V$ and $g: A \times V \rightarrow U$ then

$$
\left\langle A,\left(\left\langle\pi_{1}, g\right\rangle ; f\right)^{\dagger}\right\rangle ; g=\left(\left\langle\pi_{1}, f\right\rangle ; g\right)^{\dagger}: A \rightarrow U .
$$

There are a number of sets of axioms equivalent to Definition 2.7 which can be gleaned from [4], see also [16]. We mention in particular the axiomatization which consists of naturality, parameterized dinaturality and the diagonal property. 

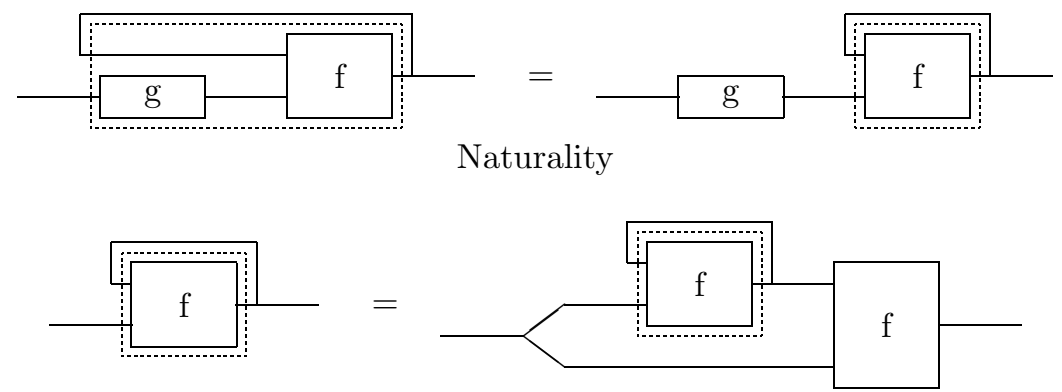

Fixed Point Property

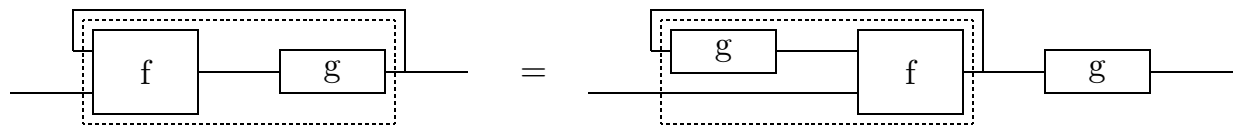

Dinaturality

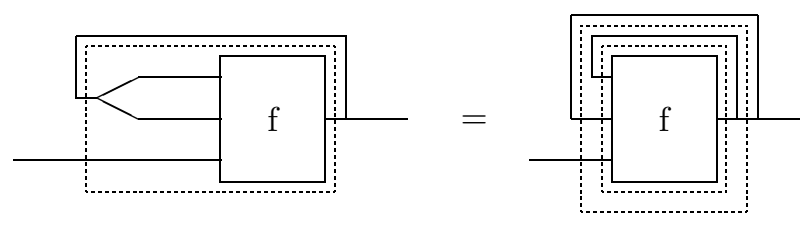

Diagonal Property

Figure 2. Conway axioms.

One focus of other work is the complete axiomatization of identities holding in special classes of models. Conway operators (Conway theories) are a stepping stone on the way. Adding the commutative identities of [4] produces a complete axiomatization, inter alia, for the least fixpoint operator in domains. These apparently mysterious identities follow most usefully from "uniformity properties" [4,11,12]. Simpson and Plotkin [32] explain the generality of the approach via uniformity, which also has other potential uses [17]. We, however, do not consider issues of uniformity further here.

An important theorem about traces and fixpoints is the following, which is due (independently) to Hasegawa and to Hyland, though its essential combinatorial content had been observed earlier in a slightly different context [3-5]:

Theorem 2.9. To give a trace on a Cartesian category $\mathbb{C}$ is to give a Conway operator on $\mathbb{C}$.

For us the notion of trace is paramount. It is completely general and encapsulates the idea of feedback (cyclic graphs) in computer science; and it supports the Geometry of Interaction construction which models the dynamics of proofs. 


\section{TRACES AND FIXPOINT OPERATORS ON PREMONOIDAL CATEGORIES}

So, what is an appropriate generalization of the notions of trace and fixpoint operator to the premonoidal case? We want definitions which make sense, have useful concrete instances, give the monoidal versions as special cases and lead to a generalization of Theorem 2.9.

\subsection{Symmetric PREMONOIDAL TRACES}

We start by trying to generalize the definition of trace to a centered symmetric premonoidal category $J: \mathbb{M} \rightarrow \mathbb{K}$. Although none of the conditions in Definition 2.4 are expressed in terms of tensoring arbitrary morphisms (in which case we would certainly have to re-examine them), we cannot simply leave the definition unchanged:

Proposition 3.1. A symmetric premonoidal category with a trace as defined in Definition 2.4 is actually monoidal.

Proof. Assume $f: A \rightarrow B$ and $g: A^{\prime} \rightarrow B^{\prime}$. Then

$$
\begin{aligned}
& f \ltimes g=f \otimes A^{\prime} ; B \otimes g \quad \text { (def) } \\
& =f \otimes A^{\prime} ; B \otimes\left(g ; \operatorname{tr}_{B^{\prime}, B^{\prime}}^{B^{\prime}}(\sigma)\right) \quad(\text { yank }) \\
& =f \otimes A^{\prime} ; B \otimes\left(\operatorname{tr}_{A^{\prime}, B^{\prime}}^{B^{\prime}}\left(g \otimes B^{\prime} ; \sigma\right)\right) \quad(\text { leftt }) \\
& =f \otimes A^{\prime} ; \operatorname{tr}_{B \otimes A^{\prime}, B \otimes B^{\prime}}^{B^{\prime}}\left(\alpha ; B \otimes\left(g \otimes B^{\prime} ; \sigma\right) ; \alpha^{-1}\right) \quad \text { (super) } \\
& =\operatorname{tr}_{A \otimes A^{\prime}, B \otimes B^{\prime}}^{B^{\prime}}\left(\left(\left(f \otimes A^{\prime}\right) \otimes B^{\prime}\right) ; \alpha ; B \otimes\left(g \otimes B^{\prime} ; \sigma\right) ; \alpha^{-1}\right) \quad \text { (leftt) } \\
& =\operatorname{tr}_{A \otimes A^{\prime}, B \otimes B^{\prime}}^{B^{\prime}}\left(\left(f \otimes A^{\prime}\right) \otimes B^{\prime} ; \alpha ; B \otimes \sigma ; \alpha^{-1} ;\left(B \otimes B^{\prime}\right) \otimes g\right) \quad \text { (struct) } \\
& =\operatorname{tr}_{A \otimes A^{\prime}, B \otimes B^{\prime}}^{A^{\prime}}\left(\left(A \otimes A^{\prime}\right) \otimes g ;\left(f \otimes A^{\prime}\right) \otimes B^{\prime} ; \alpha ; B \otimes \sigma ; \alpha^{-1}\right) \quad \text { (slide) } \\
& =\operatorname{tr}_{A \otimes A^{\prime}, B \otimes B^{\prime}}^{A^{\prime}}\left(\left(A \otimes A^{\prime}\right) \otimes g ; \alpha ; A \otimes \sigma ; \alpha^{-1} ;\left(f \otimes B^{\prime}\right) \otimes A^{\prime}\right) \quad \text { (struct) } \\
& =\operatorname{tr}_{A \otimes A^{\prime}, A \otimes B^{\prime}}^{A^{\prime}}\left(\left(A \otimes A^{\prime}\right) \otimes g ; \alpha ; A \otimes \sigma ; \alpha^{-1}\right) ; f \otimes B^{\prime} \quad \text { (rightt) } \\
& =\operatorname{tr}_{A \otimes A^{\prime}, A \otimes B^{\prime}}^{A^{\prime}}\left(\alpha ; A \otimes\left(\sigma ;\left(g \otimes A^{\prime}\right)\right) ; \alpha^{-1}\right) ; f \otimes B^{\prime} \quad \text { (struct) } \\
& =A \otimes \operatorname{tr}_{A^{\prime}, B^{\prime}}^{A^{\prime}}\left(\sigma ; g \otimes A^{\prime}\right) ; f \otimes B^{\prime} \quad \text { (super) } \\
& =A \otimes\left(\operatorname{tr}_{A^{\prime}, A^{\prime}}^{A^{\prime}}(\sigma) ; g\right) ; f \otimes B^{\prime} \quad \text { (rightt) } \\
& =A \otimes g ; f \otimes B^{\prime} \quad \text { (yank) } \\
& =f \rtimes g \quad(\text { def }) \text {. }
\end{aligned}
$$

The key step in the above proof uses the Sliding axiom to commute the side-effects of two computations. This observation motivates the following definition: 
Definition 3.2. A trace on a centered symmetric premonoidal category $J: \mathbb{M} \rightarrow$ $\mathbb{K}$ is a family of functions

$$
\operatorname{tr}_{A, B}^{U}: \mathbb{K}(A \otimes U, B \otimes U) \rightarrow \mathbb{K}(A, B)
$$

satisfying the same conditions given in Definition 2.4 except that the Sliding axiom is replaced by

Central Sliding. If $f: A \otimes U \rightarrow B \otimes V$ and if $g: V \rightarrow U$ is a central morphism then

$$
\operatorname{tr}_{A, B}^{U}(f ;(B \otimes g))=\operatorname{tr}_{A, B}^{V}((A \otimes g) ; f): A \rightarrow B
$$

and we impose the further requirement

Centre Preservation. If $f: A \otimes U \rightarrow B \otimes U$ is central then so is $\operatorname{tr}_{A, B}^{U} f$ : $A \rightarrow B$.

The axioms of a premonoidal trace are shown in Figure 3, where we follow Jeffrey $[20]$ in using a heavy line to indicate the sequencing of effects in $\mathbb{K}$ (and that line runs outside those boxes intended to represent central morphisms). Clearly, if $J: \mathbb{M} \rightarrow \mathbb{K}$ has a premonoidal trace on $\mathbb{K}$, the restriction of that trace to $\mathbb{M}$ is a trace operator in the traditional sense of Definition 2.4. In particular, Definition 3.2 really is a generalization of Definition 2.4.

Requiring the trace to preserve the distinguished centre $\mathbb{M}$ is largely a matter of taste: we prefer to keep our equations algebraic. Even without the condition it is still easy to see that the trace preserves $Z(\mathbb{K})$ :

Proposition 3.3. If $f: A \otimes U \rightarrow B \otimes U$ is in $Z(\mathbb{K})$ and $g: C \rightarrow D$ then $g \ltimes \operatorname{tr}_{A, B}^{U}(f)=g \rtimes \operatorname{tr}_{A, B}^{U}(f)$.

Proof.

$$
\begin{aligned}
g \ltimes \operatorname{tr}_{A, B}^{U}(f) & =g \otimes A ; D \otimes \operatorname{tr}_{A, B}^{U}(f) \\
& =g \otimes A ; \operatorname{tr}_{D \otimes A, D \otimes B}^{U}(D \otimes f) \quad \text { (super) } \\
& =\operatorname{tr}_{C \otimes A, D \otimes B}^{U}(g \otimes A \otimes U ; D \otimes f) \quad \text { (leftt) } \\
& =\operatorname{tr}_{C \otimes A, D \otimes B}^{U}(C \otimes f ; g \otimes B \otimes U) \quad \text { (centrality) } \\
& =\operatorname{tr}_{C \otimes A, C \otimes B}^{U}(C \otimes f) ; g \otimes B \quad \text { (rightt) } \\
& =C \otimes \operatorname{tr}_{A, B}^{U}(f) ; g \otimes B \quad \text { (super) } \\
& =g \rtimes \operatorname{tr}_{A, B}^{U}(f) .
\end{aligned}
$$

Thus there is an obvious (non-algebraic) notion of traced premonoidal category. If $\mathbb{K}$ is such and we choose $J: \mathbb{M} \rightarrow \mathbb{K}$ giving a centered premonoidal category then we may fail to get a trace on $J: \mathbb{M} \rightarrow \mathbb{K}$ since $\mathbb{M}$ may fail to be closed under trace (though one can then just close it). 


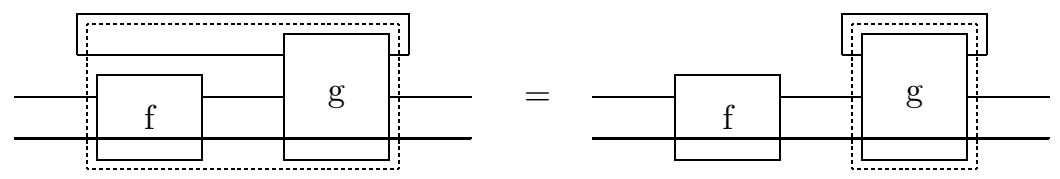

Left Tightening

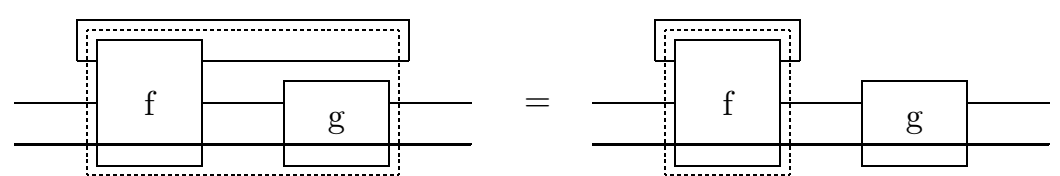

Right Tightening

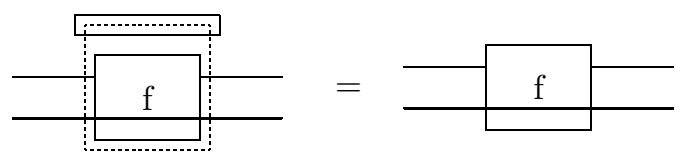

Vanishing (nullary)

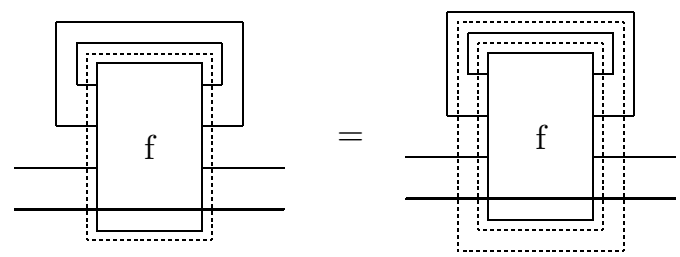

Vanishing (binary)

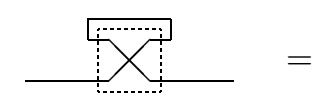

Yanking

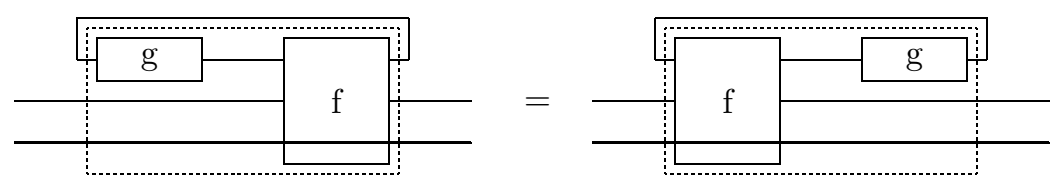

Central Sliding

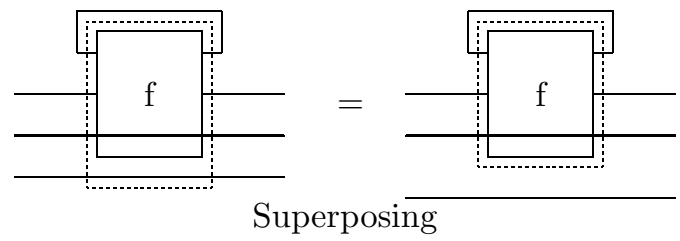

Figure 3. Premonoidal trace axioms. 
It might also be remarked that the premonoidal sliding condition appears somewhat asymmetric, since it requires that $g$, rather than one of $f$ and $g$, be central. However, a little calculation shows that the symmetric case is a consequence:

Proposition 3.4. Assume $f: A \otimes U \rightarrow B \otimes V$ is central and $g: V \rightarrow U$, then $\operatorname{tr}_{A, B}^{V}((A \otimes g) ; f)=\operatorname{tr}_{A, B}^{U}(f ; B \otimes g)$.

Proof.

$$
\begin{aligned}
& \operatorname{tr}_{A, B}^{V}((A \otimes g) ; f)=\operatorname{tr}_{A, B}^{V}((A \otimes g) ; f) ; \operatorname{tr}_{B, B}^{B}(\sigma) \quad(\text { yank }) \\
& =\operatorname{tr}_{A, B}^{B}\left(\operatorname{tr}_{A, B}^{V}((A \otimes g) ; f) \otimes B ; \sigma\right) \quad \text { (rightt) } \\
& =\operatorname{tr}_{A, B}^{B}\left(\sigma ; B \otimes \operatorname{tr}_{A, B}^{V}((A \otimes g) ; f)\right) \quad \text { (nat) } \\
& =\operatorname{tr}_{A, B}^{B}\left(\sigma ; \operatorname{tr}_{B \otimes A, B \otimes B}^{V}\left(\alpha ; B \otimes((A \otimes g) ; f) ; \alpha^{-1}\right)\right) \quad \text { (super) } \\
& =\operatorname{tr}_{A, B}^{B}\left(\operatorname{tr}_{A \otimes B, B \otimes B}^{V}\left(\sigma \otimes V ; \alpha ; B \otimes((A \otimes g) ; f) ; \alpha^{-1}\right)\right) \quad \text { (leftt) } \\
& =\operatorname{tr}_{A, B}^{B \otimes V}\left(\alpha^{-1} ; \sigma \otimes V ; \alpha ; B \otimes(A \otimes g) ; B \otimes f\right) \quad \text { (action) } \\
& =\operatorname{tr}_{A, B}^{A \otimes U}\left(A \otimes f ; \alpha^{-1} ; \sigma \otimes V ; \alpha ; B \otimes(A \otimes g)\right) \\
& \text { (premon sliding, as } B \otimes f \text { is central) } \\
& =\operatorname{tr}_{A, B}^{A \otimes U}(A \otimes(f ;(B \otimes g)) ; \sigma ; \alpha ; B \otimes \sigma) \quad \text { (struct) } \\
& =\operatorname{tr}_{A, B}^{A}\left(\operatorname{tr}_{A \otimes A, B \otimes A}^{U}\left(\alpha ; A \otimes(f ; B \otimes g) ; \sigma ; \alpha ; B \otimes \sigma ; \alpha^{-1}\right)\right) \text { (action) } \\
& =\operatorname{tr}_{A, B}^{A}\left(\operatorname{tr}_{A \otimes A, B \otimes A}^{U}\left(\alpha ; A \otimes(f ; B \otimes g) ; \alpha^{-1} ; \sigma \otimes U\right)\right) \quad \text { (struct) } \\
& =\operatorname{tr}_{A, B}^{A}\left(\operatorname{tr}_{A \otimes A, A \otimes B}^{U}\left(\alpha ; A \otimes(f ; B \otimes g) ; \alpha^{-1}\right) ; \sigma\right) \quad \text { (rightt) } \\
& =\operatorname{tr}_{A, B}^{A}\left(A \otimes \operatorname{tr}_{A, B}^{U}(f ; B \otimes g) ; \sigma\right) \quad \text { (super) } \\
& =\operatorname{tr}_{A, B}^{A}\left(\sigma ; \operatorname{tr}_{A, B}^{U}(f ; B \otimes g) \otimes A\right) \quad \text { (nat) } \\
& =\operatorname{tr}_{A, A}^{A}(\sigma) ; \operatorname{tr}_{A, B}^{U}(f ; B \otimes g) \quad \text { (leftt) } \\
& =\operatorname{tr}_{A, B}^{U}(f ; B \otimes g) \quad \text { (yank). }
\end{aligned}
$$

\subsection{Symmetric PREMONOIDAL FIXPOINTS}

We now turn to generalizing the notion of fixpoint operator to the premonoidal case. Since some of the axioms involve duplication and discarding, we will assume that we are working in a Freyd category $J: \mathbb{C} \rightarrow \mathbb{K}$. We also use $\Delta, \pi_{1},\langle\cdot, \cdot\rangle$, etc. as shorthand notation for the lifting of the appropriate operations from $\mathbb{C}$ to $\mathbb{K}$ (i.e. we elide uses of $J$ ). The notation $\langle f, g\rangle$ is ambiguous unless we specify the order in which the components are computed, but we shall only use it in the case one of the maps is central. 
Definition 3.5. A parameterized fixpoint operator on a Freyd category $J: \mathbb{C} \rightarrow \mathbb{K}$ is a family of functions

$$
(\cdot)^{*}: \mathbb{K}(A \otimes U, U) \rightarrow \mathbb{K}(A, U)
$$

which satisfies:

Centre Preservation. If $f: A \otimes U \rightarrow U$ is central then so is $f^{*}: A \rightarrow U$. Naturality. If $f: B \otimes U \rightarrow U$ and $g: A \rightarrow B$ then

$$
g ; f^{*}=((g \otimes U) ; f)^{*}: A \rightarrow U .
$$

Central Fixed Point Property. If $f: A \otimes U \rightarrow U$ is central, then

$$
\left\langle A, f^{*}\right\rangle ; f=f^{*}: A \rightarrow U \text {. }
$$

Just as in the Cartesian case, this is the bare minimum one might require of a fixpoint operator. We are interested in rather stronger conditions, and propose the following as an appropriate generalization of Conway operators on Cartesian categories:

Definition 3.6. A parameterized fixpoint operator $(\cdot)^{*}$ on a Freyd category is a Conway operator if it satisfies the following conditions.

Parallel Property. If $f: A \otimes U \rightarrow U$ and $g: B \otimes V \rightarrow V$ with one of $f$ and $g$ central then

$$
(A \otimes \sigma \otimes V ; f \otimes g)^{*}=f^{*} \otimes g^{*}: A \otimes B \rightarrow U \otimes V .
$$

Withering Property. If $f: A \otimes U \rightarrow B \otimes U$ and $g: B \rightarrow C$ then

$$
\left(\left\langle\pi_{1}, \pi_{3}\right\rangle ; f ; g \otimes U\right)^{*}=\left(\left\langle\pi_{1}, \pi_{3}\right\rangle ; f\right)^{*} ; g \otimes U: A \rightarrow C \otimes U .
$$

Diagonal Property. If $f: A \otimes U \otimes U \rightarrow U$ then

$$
((A \otimes \Delta) ; f)^{*}=\left(f^{*}\right)^{*}: A \rightarrow U .
$$

Dinaturality. If $f: A \otimes U \rightarrow V$ and $g: V \rightarrow U$ with $g$ central then

$$
(f ; g)^{*}=(A \otimes g ; f)^{*} ; g: A \rightarrow U .
$$

The axioms of a premonoidal Conway operator are shown graphically in Figure 4 . Dinaturality and the diagonal property are essentially the same as in the Cartesian 
case, but the parallel and withering properties are more unusual. There is a natural generalization of parameterized dinaturality to Freyd categories:

Definition 3.7. A parameterized fixpoint operator $(\cdot)^{*}$ on a Freyd category satisfies parameterized central dinaturality if, given $f: A \otimes U \rightarrow V$ and $g: A \otimes V \rightarrow U$ with $g$ central

$$
\left(\left\langle\pi_{1}, f\right\rangle ; g\right)^{*}=\left\langle A,\left(\left\langle\pi_{1}, g\right\rangle ; f\right)^{*}\right\rangle ; g .
$$

As in the Cartesian case, parameterized central dinaturality clearly implies the central fixed point property. But in the case of Freyd categories, parameterized dinaturality does not seem sufficient (along with the diagonal property) to establish the equivalence between traces and Conway operators, which is what motivated our parallel and withering axioms. These do imply parameterized dinaturality, however, though we defer the formal statement of this (Prop. 3.15) as our proof uses the trace axioms.

Furthermore, our definition of a Conway operator on a Freyd category does generalize the standard one:

Proposition 3.8. Definition 3.6 is equivalent to Definition 2.7 in the case that the category is Cartesian.

\subsection{RELATING FIXPOINTS AND TRACES IN FREYD CATEGORIES}

We now show our main result: in a Freyd category, to give a premonoidal trace is equivalent to giving a premonoidal Conway operator.

Theorem 3.9. Let $J: \mathbb{C} \rightarrow \mathbb{K}$ be a Freyd category such that $\mathbb{K}$ is traced, as in Definition 3.2. Then the operation

$$
(\cdot)^{*}: \mathbb{K}(A \otimes U, U) \rightarrow \mathbb{K}(A, U)
$$

defined by, for $f: A \otimes U \rightarrow U:$

$$
f^{*}=\operatorname{tr}_{A, U}^{U}(f ; \Delta)
$$

is a Conway operator in the sense of Definition 3.6.

Proof. Naturality and centre preservation are immediate. 


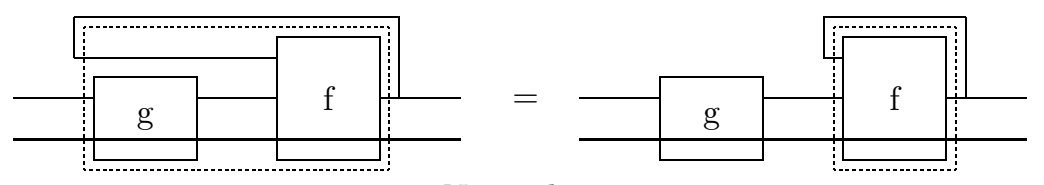

Naturality

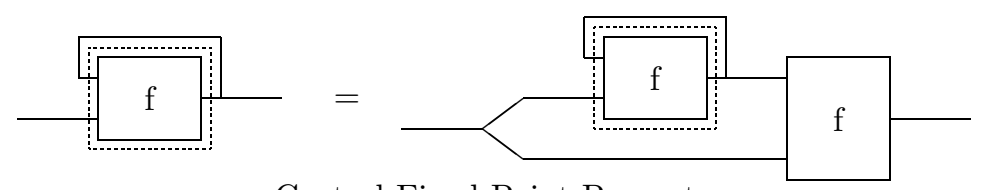

Central Fixed Point Property

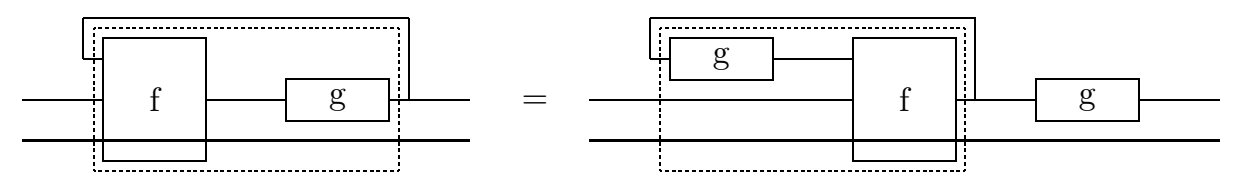

Central Dinaturality

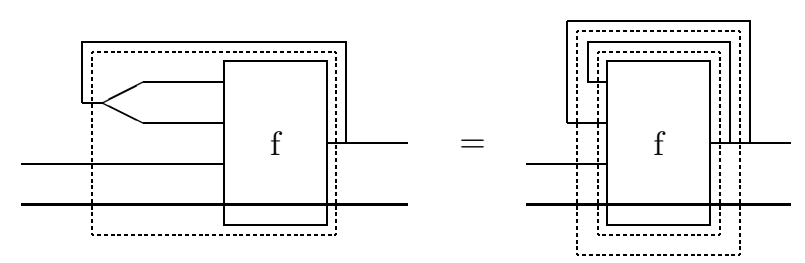

Diagonal Property
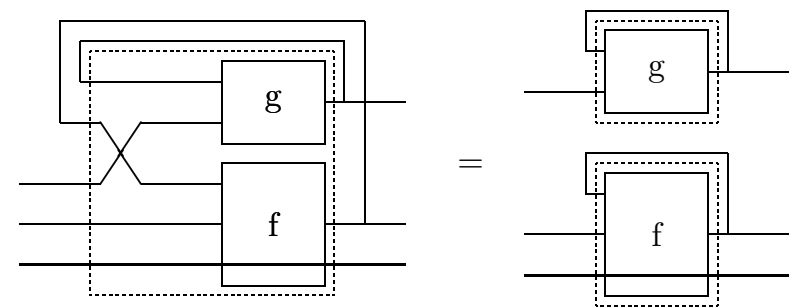

Parallel Property
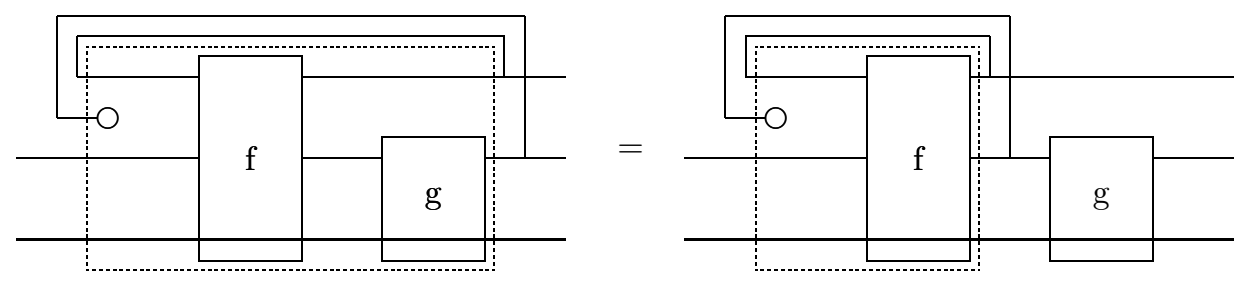

Withering Property

Figure 4. Premonoidal conway axioms. 
For the central fixed point property, assume that $f: A \otimes U \rightarrow U$ is central. Then

$$
\begin{aligned}
\left\langle A, f^{*}\right\rangle ; f & =\Delta ; A \otimes \operatorname{tr}_{A, U}^{U}(f ; \Delta) ; f \\
& =\Delta ; A \otimes\left(\operatorname{tr}_{A, A}^{A}(\sigma) ; \operatorname{tr}_{A, U}^{U}(f ; \Delta)\right) ; f \quad \text { (yank) } \\
& =\Delta ; A \otimes\left(\operatorname{tr}_{A, U}^{A}\left(\sigma ; \operatorname{tr}_{A, U}^{U}(f ; \Delta) \otimes A\right)\right) ; f \quad \text { (rightt) } \\
& =\Delta ; A \otimes\left(\operatorname{tr}_{A, U}^{A}\left(A \otimes \operatorname{tr}_{A, U}^{U}(f ; \Delta) ; \sigma\right)\right) ; f \\
& =\Delta ; A \otimes\left(\operatorname{tr}_{A, U}^{A}\left(\operatorname{tr}_{A \otimes A, A \otimes U}^{U}(A \otimes f ; A \otimes \Delta) ; \sigma\right)\right) ; f \quad \text { (super) } \\
& =\Delta ; A \otimes\left(\operatorname{tr}_{A, U}^{A}\left(\operatorname{tr}_{A \otimes A, A \otimes U}^{U}(A \otimes f ; A \otimes \Delta ; \sigma \otimes U)\right) ; f \quad\right. \text { (rightt) } \\
& =\Delta ; A \otimes\left(\operatorname{tr}_{A, U}^{A \otimes U}(A \otimes f ; A \otimes \Delta ; \sigma \otimes U)\right) ; f \quad(\text { action }) \\
& =\Delta ; A \otimes\left(\operatorname{tr}_{A, U}^{U}(A \otimes \Delta ; \sigma \otimes U ; U \otimes f)\right) ; f \quad(\text { slide }) \\
& =\Delta ; \operatorname{tr}_{A \otimes A, A \otimes U}^{U}(A \otimes A \otimes \Delta ; A \otimes \sigma \otimes U ; A \otimes U \otimes f) ; f \quad \text { (super) } \\
& =\Delta ; \operatorname{tr}_{A \otimes A, U}^{U}(A \otimes A \otimes \Delta ; A \otimes \sigma \otimes U ; A \otimes U \otimes f ; f \otimes U) \quad \text { (rightt) } \\
& =\operatorname{tr}_{A, U}^{U}(\Delta \otimes U ; A \otimes A \otimes \Delta ; A \otimes \sigma \otimes U ; A \otimes U \otimes f ; f \otimes U) \quad \text { (leftt) } \\
& =\operatorname{tr}_{A, U}^{U}(\Delta ; f \otimes f) \quad(f \text { central }) \\
& =\operatorname{tr}_{A, U}^{U}(f ; \Delta) \\
& =f^{*} .
\end{aligned}
$$

For central dinaturality, assume $f: A \otimes U \rightarrow V$ and $g: V \rightarrow U$ with $g$ central. Then

$$
\begin{aligned}
(f ; g)^{*} & =\operatorname{tr}_{A, U}^{U}(f ; g ; \Delta) \\
& =\operatorname{tr}_{A, U}^{U}(f ; \Delta ; g \otimes V ; U \otimes g) \quad(g \text { central }) \\
& =\operatorname{tr}_{A, U}^{V}(A \otimes g ; f ; \Delta ; g \otimes V) \quad \text { (sliding) } \\
& =\operatorname{tr}_{A, U}^{V}(A \otimes g ; f ; \Delta) ; g \quad \text { (rightt) } \\
& =(A \otimes g ; f)^{*} ; g .
\end{aligned}
$$

For the diagonal property, assume $f: A \otimes U \otimes U \rightarrow U$, then

$$
\begin{aligned}
\left(f^{*}\right)^{*} & =\operatorname{tr}_{A, U}^{U}\left(\operatorname{tr}_{A \otimes U, U}^{U}(f ; \Delta) ; \Delta\right) \\
& =\operatorname{tr}_{A, U}^{U}\left(\operatorname{tr}_{A \otimes U, U \otimes U}^{U}(f ; \Delta ; \Delta \otimes U)\right) \quad \text { (rightt) } \\
& =\operatorname{tr}_{A, U}^{U \otimes U}(f ; \Delta ; \Delta \otimes U) \quad \text { (action) } \\
& =\operatorname{tr}_{A, U}^{U \otimes U}(f ; \Delta ; U \otimes \Delta) \quad \text { (struct) } \\
& =\operatorname{tr}_{A, U}^{U}(A \otimes \Delta ; f ; \Delta) \quad \text { (slide, } \Delta \text { central) } \\
& =(A \otimes \Delta ; f)^{*} .
\end{aligned}
$$


To show the withering property, assume $f: A \otimes U \rightarrow B \otimes U$ and $g: B \rightarrow C$. Then

$$
\begin{aligned}
\left(\left\langle\pi_{1}, \pi_{3}\right\rangle ; f ; g \otimes U\right)^{*} & =\operatorname{tr}_{A, C \otimes U}^{C \otimes U}\left(A \otimes \pi_{2} ; f ; g \otimes U ; \Delta\right) \\
& =\operatorname{tr}_{A, C \otimes U}^{U}\left(f ; g \otimes U ; \Delta ; C \otimes U \otimes \pi_{2}\right) \quad \text { (slide) } \\
& =\operatorname{tr}_{A, C \otimes U}^{U}(f ; g \otimes U ; C \otimes \Delta) \\
& =\operatorname{tr}_{A, C \otimes U}^{U}(f ; B \otimes \Delta ; g \otimes U \otimes U) \\
& =\operatorname{tr}_{A, B \otimes U}^{U}(f ; B \otimes \Delta) ; g \otimes U \quad \text { (rightt) } \\
& =\operatorname{tr}_{A, B \otimes U}^{U}\left(f ; \Delta ; B \otimes U \otimes \pi_{2}\right) ; g \otimes U \\
& =\operatorname{tr}_{A, B \otimes U}^{B \otimes U}\left(A \otimes \pi_{2} ; f ; \Delta\right) ; g \otimes U \quad \text { (slide) } \\
& =\left(\left\langle\pi_{1}, \pi_{3}\right\rangle ; f\right)^{*} ; g \otimes U .
\end{aligned}
$$

To show the parallel property, assume $f: A \otimes U \rightarrow U, g: B \otimes V \rightarrow V$ and, without loss of generality, that $f$ is central. Then

$$
\begin{aligned}
& (A \otimes \sigma \otimes V ; f \otimes g)^{*} \\
= & \operatorname{tr}_{A \otimes B, U \otimes V}^{U \otimes V}(A \otimes \sigma \otimes V ; f \otimes g ; \Delta) \\
= & \operatorname{tr}_{A \otimes B, U \otimes V}^{U}\left(\operatorname{tr}_{A \otimes B \otimes U, U \otimes V \otimes U}^{V}(A \otimes \sigma \otimes V ; f \otimes g ; \Delta)\right) \quad \text { (action) } \\
= & \operatorname{tr}_{A \otimes B, U \otimes V}^{U}\left(A \otimes \sigma ; \operatorname{tr}_{A \otimes U \otimes B, U \otimes V \otimes U}^{V}(f \otimes B \otimes V ; U \otimes g ; \Delta)\right) \quad \text { (leftt) } \\
= & \operatorname{tr}_{A \otimes B, U \otimes V}^{U}\left(A \otimes \sigma ; f \otimes B ; \operatorname{tr}_{U \otimes B, U \otimes V \otimes U}^{V}(U \otimes g ; \Delta)\right) \quad(\text { leftt }) \\
= & \operatorname{tr}_{A \otimes B, U \otimes V}^{U}\left(A \otimes \sigma ; f \otimes B ; \operatorname{tr}_{U \otimes B, U \otimes V \otimes U}^{V}(U \otimes g ; \Delta \otimes \Delta ; U \otimes \sigma \otimes V)\right) \\
= & \operatorname{tr}_{A \otimes B, U \otimes V}^{U}\left(A \otimes \sigma ; f \otimes B ; \operatorname{tr}_{U \otimes B, U \otimes U \otimes V}^{V}(U \otimes g ; \Delta \otimes \Delta) ; U \otimes \sigma\right) \quad(\text { rightt }) \\
= & \operatorname{tr}_{A \otimes B, U \otimes V}^{U}\left(A \otimes \sigma ; f \otimes B ; \operatorname{tr}_{U \otimes B, U^{2} \otimes V}^{V}\left(\Delta \otimes B \otimes V ; U^{2} \otimes(g ; \Delta)\right) ; U \otimes \sigma\right) \\
= & \operatorname{tr}_{A \otimes B, U \otimes V}^{U}\left(A \otimes \sigma ; f \otimes B ; \Delta \otimes B ; \operatorname{tr}_{U}^{V} \otimes B, U^{2} \otimes V\right. \\
& (\operatorname{leftt}) \\
= & \left.\operatorname{tr}_{A \otimes B, U \otimes V}^{U}(A \otimes U \otimes(g ; \Delta)) ; U \otimes \sigma\right) \\
= & \operatorname{tr}_{A \otimes B, U \otimes V}^{U}\left(A \otimes \sigma ; f \otimes B ; \Delta \otimes B ; U \otimes \sigma ; U \otimes \operatorname{tr}_{B, V}^{V}(g ; \Delta) \otimes U\right) \\
= & \operatorname{tr}_{A \otimes B, U \otimes B}^{U}(A \otimes \sigma ; f \otimes B ; \Delta \otimes B ; U \otimes \sigma) ; U \otimes \operatorname{tr}_{B, V}^{V}(g ; \Delta) \quad(\text { rightt }) \\
= & \operatorname{tr}_{A \otimes B, U \otimes B}^{U}(\sigma \otimes U ; B \otimes(f ; \Delta) ; \sigma \otimes U) ; U \otimes \operatorname{tr}_{B, V}^{V}(g ; \Delta) \\
= & \sigma ; \operatorname{tr}_{B \otimes A, B \otimes U}^{U}(B \otimes(f ; \Delta)) ; \sigma ; U \otimes \operatorname{tr}_{B, V}^{V}(g ; \Delta) \quad(\mathrm{l} / \mathrm{r} \mathrm{t}) \\
= & \sigma ; B \otimes \operatorname{tr}_{A, B}^{U}(f ; \Delta) ; \sigma ; U \otimes \operatorname{tr}_{B, V}^{V}(g ; \Delta) \quad(\operatorname{super}) \\
= & \operatorname{tr}_{A, B}^{U}(f ; \Delta) \otimes B ; U \otimes \operatorname{tr}_{B, V}^{V}(g ; \Delta) \\
= & f^{*} \otimes g^{*} .
\end{aligned}
$$

Remark 3.10. Hasegawa has also given a construction for a fixpoint operator from a trace in the special case of the Kleisli category of a commutative strong monad on a Cartesian category (a case in which the premonoidal structure is 
monoidal) [16, Th. 7.2.1]. However, restricting our construction to this special case does not generally give the same fixpoint operator. Hasegawa's construction uses the adjunction in an essential way and repeats side-effects.

Theorem 3.11. Let $J: \mathbb{C} \rightarrow \mathbb{K}$ be a Freyd category where $\mathbb{K}$ has a Conway operator $(\cdot)^{*}$ as defined in Definition 3.6. Then the operation

$$
\operatorname{tr}_{A, B}^{U}(\cdot): \mathbb{K}(A \otimes U, B \otimes U) \rightarrow \mathbb{K}(A, B)
$$

defined by, for $f: A \otimes U \rightarrow B \otimes U$

$$
\operatorname{tr}_{A, B}^{U}(f)=\left(\left\langle\pi_{1}, \pi_{3}\right\rangle ; f\right)^{*} ; \pi_{1}: A \rightarrow B
$$

is a premonoidal trace in the sense of Definition 3.2.

Proof. Centre preservation is immediate, and left tightening follows directly from naturality.

For right tightening, let $f: A \otimes U \rightarrow B \otimes U$ and $g: B \rightarrow C$. Then

$$
\begin{aligned}
\operatorname{tr}_{A, C}^{U}(f ; g \otimes U) & =\left(\left\langle\pi_{1}, \pi_{3}\right\rangle ; f ; g \otimes U\right)^{*} ; \pi_{1} \\
& =\left(\left\langle\pi_{1}, \pi_{3}\right\rangle ; f ;\right)^{*} ; g \otimes U ; \pi_{1} \quad \text { (wither) } \\
& =\left(\left\langle\pi_{1}, \pi_{3}\right\rangle ; f ;\right)^{*} ; \pi_{1} ; g \\
& =\operatorname{tr}_{A, B}^{U}(f) ; g .
\end{aligned}
$$

For yanking, observe that $\left\langle\pi_{1}, \pi_{3}\right\rangle ; \sigma$ is central, then

$$
\begin{aligned}
\operatorname{tr}_{U, U}^{U}(\sigma) & =\left(\left\langle\pi_{1}, \pi_{3}\right\rangle ; \sigma\right)^{*} ; \pi_{1} \\
& =\left\langle U,\left(\left\langle\pi_{1}, \pi_{3}\right\rangle ; \sigma\right)^{*}\right\rangle ;\left\langle\pi_{1}, \pi_{3}\right\rangle ; \sigma ; \pi_{1} \quad(\mathrm{cfpp}) \\
& =\left\langle U,\left(\left\langle\pi_{1}, \pi_{3}\right\rangle ; \sigma\right)^{*}\right\rangle ; \pi_{3} \\
& =\left(\left\langle\pi_{1}, \pi_{3}\right\rangle ; \sigma\right)^{*} ; \pi_{2} \\
& =\left\langle U,\left(\left\langle\pi_{1}, \pi_{3}\right\rangle ; \sigma\right)^{*}\right\rangle ;\left\langle\pi_{1}, \pi_{3}\right\rangle ; \sigma ; \pi_{2} \quad(\mathrm{cfpp}) \\
& =\left\langle U,\left(\left\langle\pi_{1}, \pi_{3}\right\rangle ; \sigma\right)^{*}\right\rangle ; \pi_{1} \\
& =U
\end{aligned}
$$

For the nullary case of action, assume $f: A \rightarrow B$ then

$$
\begin{aligned}
\operatorname{tr}_{A, B}^{I}(f \otimes I) & =\left(\left\langle\pi_{1}, \pi_{3}\right\rangle ; f \otimes I\right)^{*} ; \pi_{1} \\
& \left.=\left\langle\pi_{1}, \pi_{3}\right\rangle^{*} ; f \otimes I ; \pi_{1} \quad \text { (wither }\right) \\
& =\left\langle A,\left\langle\pi_{1}, \pi_{3}\right\rangle^{*}\right\rangle ;\left\langle\pi_{1}, \pi_{3}\right\rangle ; f \otimes I ; \pi_{1} \quad(\text { cfpp }) \\
& =\left\langle f,\left\langle\pi_{1}, \pi_{3}\right\rangle^{*} ; \pi_{2}\right\rangle ; \pi_{1} \\
& =f .
\end{aligned}
$$


For the binary case of action, assume $f: A \otimes U \otimes V \rightarrow B \otimes U \otimes V$, then

$$
\begin{aligned}
\operatorname{tr}_{A, B}^{U}\left(\operatorname{tr}_{A \otimes U, B \otimes U}^{V}(f)\right) & =\left(\left\langle\pi_{1}, \pi_{3}\right\rangle ;\left(\left\langle\pi_{1}, \pi_{2}, \pi_{5}\right\rangle ; f\right)^{*} ;\left\langle\pi_{1}, \pi_{2}\right\rangle\right)^{*} ; \pi_{1} \\
& =\left(A \otimes\left\langle\pi_{1}, \pi_{2}\right\rangle ;\left\langle\pi_{1}, \pi_{3}\right\rangle ;\left(\left\langle\pi_{1}, \pi_{2}, \pi_{5}\right\rangle ; f\right)^{*}\right)^{*} ;\left\langle\pi_{1}, \pi_{2}\right\rangle ; \pi_{1} \\
& \quad \text { dinaturality) } \\
& =\left(\left\langle\pi_{1}, \pi_{3}\right\rangle ;\left(\left\langle\pi_{1}, \pi_{2}, \pi_{5}\right\rangle ; f\right)^{*}\right)^{*} ; \pi_{1} \quad \\
& =\left(\left(\left\langle\pi_{1}, \pi_{3}, \pi_{7}\right\rangle ; f\right)^{*}\right)^{*} ; \pi_{1} \quad \text { (naturality) } \\
& =\left(A \otimes \Delta ;\left\langle\pi_{1}, \pi_{3}, \pi_{7}\right\rangle ; f\right)^{*} ; \pi_{1} \quad \text { (diagonal) } \\
& =\left(\left\langle\pi_{1}, \pi_{3}, \pi_{4}\right\rangle ; f\right)^{*} ; \pi_{1} \\
& =\operatorname{tr}_{A, B}^{U \otimes V}(f) .
\end{aligned}
$$

To show sliding, let $f: A \otimes U \rightarrow B \otimes V$ and $g: V \rightarrow U$ with $g$ central. Then

$$
\begin{aligned}
\operatorname{tr}_{A, B}^{U}(f ;(B \otimes g)) & =\left(\left\langle\pi_{1}, \pi_{3}\right\rangle ; f ; B \otimes g\right)^{*} ; \pi_{1} \\
& =\left(A \otimes B \otimes g ;\left\langle\pi_{1}, \pi_{3}\right\rangle ; h\right)^{*} ; B \otimes g ; \pi_{1} \quad \text { (dinaturality) } \\
& =\left(A \otimes B \otimes g ;\left\langle\pi_{1}, \pi_{3}\right\rangle ; h\right)^{*} ; \pi_{1} \quad \text { (g central) } \\
& =\left(\left\langle\pi_{1}, \pi_{3}\right\rangle ; A \otimes g ; h\right)^{*} ; \pi_{1} \\
& =\operatorname{tr}_{A, B}^{V}((A \otimes g) ; f) .
\end{aligned}
$$

For superposing, assume $f: A \otimes U \rightarrow B \otimes U$ then

$$
\begin{aligned}
\operatorname{tr}_{C \otimes A, C \otimes B}^{U}(f) & =\left(\left\langle\pi_{1}, \pi_{2}, \pi_{5}\right\rangle ; C \otimes f\right)^{*} ;\left\langle\pi_{1}, \pi_{2}\right\rangle \\
& =\left(C \otimes \sigma \otimes B \otimes U ;\left\langle\pi_{1}, \pi_{3}, \pi_{5}\right\rangle ; C \otimes f\right)^{*} ;\left\langle\pi_{1}, \pi_{2}\right\rangle \\
& =\left(C \otimes \sigma \otimes B \otimes U ; \pi_{1} \otimes\left(\left\langle\pi_{1}, \pi_{3}\right\rangle ; f\right)\right)^{*} ;\left\langle\pi_{1}, \pi_{2}\right\rangle \\
& =\left(\pi_{1}^{*} \otimes\left(\left\langle\pi_{1}, \pi_{3}\right\rangle ; f\right)^{*}\right) ;\left\langle\pi_{1}, \pi_{2}\right\rangle \quad(\text { parallel }) \\
& =\left(\left(\left\langle C, \pi_{1}^{*}\right\rangle ; \pi_{1}\right) \otimes\left(\left\langle\pi_{1}, \pi_{3}\right\rangle ; f\right)^{*}\right) ;\left\langle\pi_{1}, \pi_{2}\right\rangle \quad(\mathrm{cfpp}) \\
& =\left(C \otimes\left(\left\langle\pi_{1}, \pi_{3}\right\rangle ; f\right)^{*}\right) ;\left\langle\pi_{1}, \pi_{2}\right\rangle \\
& =C \otimes\left(\left(\left\langle\pi_{1}, \pi_{3}\right\rangle ; f\right)^{*} ; \pi_{1}\right) \\
& =C \otimes \operatorname{tr}_{A, B}^{U}(f) .
\end{aligned}
$$

Proposition 3.12. The constructions of trace from Conway operator and of Conway operator from trace given in Theorems 3.11 and 3.9 respectively are mutually inverse.

Proof. Assume we have a premonoidal Conway operator $(\cdot)^{*}$. The Conway operator obtained from the trace obtained from $(\cdot)^{*}$ maps $f: A \otimes U \rightarrow U$ to 
$\left(\left\langle\pi_{1}, \pi_{3}\right\rangle ; f ; \Delta\right)^{*} ; \pi_{1}$. Then

$$
\begin{aligned}
\left(\left\langle\pi_{1}, \pi_{3}\right\rangle ; f ; \Delta\right)^{*} ; \pi_{1} & =\left(A \otimes \pi_{2} ; f ; \Delta\right)^{*} ; \pi_{1} \\
& =\left(A \otimes \Delta ; A \otimes \pi_{2} ; f\right)^{*} ; \Delta ; \pi_{1} \quad \text { (dinaturality) } \\
& =(f)^{*} ; \Delta ; \pi_{1} \\
& =(f)^{*}
\end{aligned}
$$

as required.

Going the other way around, assume we have a premonoidal trace $\operatorname{tr} \cdot$ on a Freyd category. Then we have another trace which maps $h: A \otimes U \rightarrow B \otimes U$ to $\operatorname{tr}_{A, B \otimes U}^{B \otimes U}\left(A \otimes \pi_{2} ; h ; \Delta\right) ; \pi_{1}$ and we calculate

$$
\begin{aligned}
\operatorname{tr}_{A, B \otimes U}^{B \otimes U}\left(A \otimes \pi_{2} ; h ; \Delta\right) ; \pi_{1} & =\operatorname{tr}_{A, B \otimes U}^{U}\left(h ; \Delta ; B \otimes U \otimes \pi_{2}\right) ; \pi_{1} \quad \text { (sliding) } \\
& =\operatorname{tr}_{A, B \otimes U}^{U}(h ; B \otimes \Delta) ; \pi_{1} \\
& =\operatorname{tr}_{A, B}^{U}\left(h ; B \otimes \Delta ; B \otimes \pi_{1}\right) \quad \text { (rightt) } \\
& =\operatorname{tr}_{A, B}^{U}(h) .
\end{aligned}
$$

Thus we have succeeded in establishing a premonoidal generalization of Theorem 2.9 .

Remark 3.13. Starting from a fixpoint operator, there is another candidate for the definition of a trace, viz

$$
\operatorname{tr}^{\prime}(f)=\left\langle A,\left(f ; \pi_{2}\right)^{*}\right\rangle ; f ; \pi_{1}: A \rightarrow B
$$

where $f: A \otimes U \rightarrow B \otimes U$. If $\mathbb{K}$ is monoidal this is the same as the construction used in Theorem 3.11, but in the general premonoidal case they are different, and $\operatorname{tr}^{\prime}$ does not seem to have useful properties.

Given the equivalence between traces and Conway operators in Freyd categories, we can now use the trace axioms to prove a useful lemma about Conway operators. We can then prove our earlier claim that our axioms for Conway operators imply the parameterized form of central dinaturality.

Lemma 3.14. If $h: C \otimes A \otimes U \rightarrow U$ then

$$
(A \otimes h)^{*}=\Delta \otimes C ; A \otimes \sigma ; A \otimes h .^{*}
$$


Proof.

$$
\begin{aligned}
(A \otimes h)^{*} & =\operatorname{tr}_{A \otimes C, A \otimes U}^{A \otimes U}(A \otimes h ; \Delta) \quad \text { (equivalence) } \\
& =\operatorname{tr}_{A \otimes C, A \otimes U}^{A}\left(\operatorname{tr}_{A \otimes C \otimes A, A \otimes U \otimes A}^{U}(A \otimes h ; \Delta)\right) \quad \text { (action) } \\
& =\operatorname{tr}_{A \otimes C, A \otimes U}^{A}\left(\operatorname{tr}_{A \otimes C \otimes A, A \otimes U \otimes A}^{U}(A \otimes h ; A \otimes \Delta ; \Delta \otimes U \otimes U ; A \otimes \sigma \otimes U)\right) \\
& =\operatorname{tr}_{A \otimes C, A \otimes U}^{A}\left(\operatorname{tr}_{A \otimes C \otimes A, A \otimes U}^{U}(A \otimes h ; A \otimes \Delta) ; \Delta \otimes U ; A \otimes \sigma\right) \quad \text { (rightt) } \\
& =\operatorname{tr}_{A \otimes C, A \otimes U}^{A}\left(A \otimes \operatorname{tr}_{C \otimes A, U}^{U}(h ; \Delta) ; \Delta \otimes U ; A \otimes \sigma\right) \quad \text { (super) } \\
& =\operatorname{tr}_{A \otimes C, A \otimes U}^{A}\left(A \otimes h^{*} ; \Delta \otimes U ; A \otimes \sigma\right) \quad \text { (equivalence) } \\
& =\operatorname{tr}_{A \otimes C, A \otimes U}^{A}\left(\Delta \otimes C \otimes A ; A \otimes \sigma \otimes A ; A \otimes C \otimes \sigma ; A \otimes h^{*} \otimes A\right) \\
& =\Delta \otimes C ; A \otimes \sigma ; \operatorname{tr}_{A \otimes C \otimes A, A \otimes C \otimes A}^{A}(A \otimes C \otimes \sigma) ; A \otimes h^{*} \quad \text { (lt /rt) } \\
& =\Delta \otimes C ; A \otimes \sigma ; A \otimes C \otimes \operatorname{tr}_{A, A}^{A}(\sigma) ; A \otimes h^{*} \quad(\text { super) } \\
& =\Delta \otimes C ; A \otimes \sigma ; A \otimes h^{*} \quad(\text { yank). }
\end{aligned}
$$

Proposition 3.15. A Conway operator on a Freyd category satisfies parameterized central dinaturality.

Proof. Assume $f: A \otimes U \rightarrow V$ and $g: A \otimes V \rightarrow U$ with $g$ central. Then

$$
\begin{aligned}
\left(\left\langle\pi_{1}, f\right\rangle ; g\right)^{*} & =(\Delta \otimes U ; A \otimes f ; g)^{*} \\
& =(A \otimes g ; \Delta \otimes U ; A \otimes f)^{*} ; g \quad(\text { dinaturality) } \\
& =(\Delta \otimes A \otimes V ; A \otimes A \otimes g ; A \otimes f)^{*} ; g \\
& =\Delta ;(A \otimes(A \otimes g ; f))^{*} ; g \quad(\text { naturality) } \\
& =\Delta ; \Delta \otimes A ; A \otimes \sigma ; A \otimes(A \otimes g ; f)^{*} ; g \quad \text { (Lemma 3.14) } \\
& =\Delta ; A \otimes \Delta ; A \otimes(A \otimes g ; f)^{*} ; g \\
& =\Delta ; A \otimes(\Delta \otimes V ; A \otimes g ; f)^{*} ; g \quad \text { (naturality) } \\
& =\left\langle A,\left(\left\langle\pi_{1}, g\right\rangle ; f\right)^{*}\right\rangle ; g .
\end{aligned}
$$

\section{EXAMPLES}

\subsection{MONOIDS}

Let $\mathbb{M}$ be a traced symmetric monoidal category as in Definition 2.4 and $(M, \mu$ : $M \otimes M \rightarrow M, \eta: I \rightarrow M)$ be a monoid in $\mathbb{M}$. Let $\mathbb{K}$ be the Kleisli category of the monad $T A=M \otimes A$ on $\mathbb{M}$, so $\mathbb{K}(A, B)=\mathbb{M}(A, M \otimes B)$. Then the tensor on $\mathbb{M}$ lifts so that $J: \mathbb{M} \rightarrow \mathbb{K}$ is a centered symmetric premonoidal category (it is monoidal iff $M$ is a commutative monoid). 
Proposition 4.1. In the above situation, the operation

$$
\hat{\operatorname{tr}}_{A, B}^{U}: \mathbb{K}(A \otimes U, B \otimes U) \rightarrow \mathbb{K}(A, B)
$$

defined by $\hat{\operatorname{tr}}_{A, B}^{U}(f)=\operatorname{tr}_{A, M \otimes B}^{U}(f)$ is a premonoidal trace.

Notions of computation based on monoids are fairly common. Commutative monoids such as the natural numbers under addition can be used for modeling resource usage (e.g. timed computations) whereas non-commutative monoids model, for example, side-effecting output. In Haskell syntax, the signature could look like this:

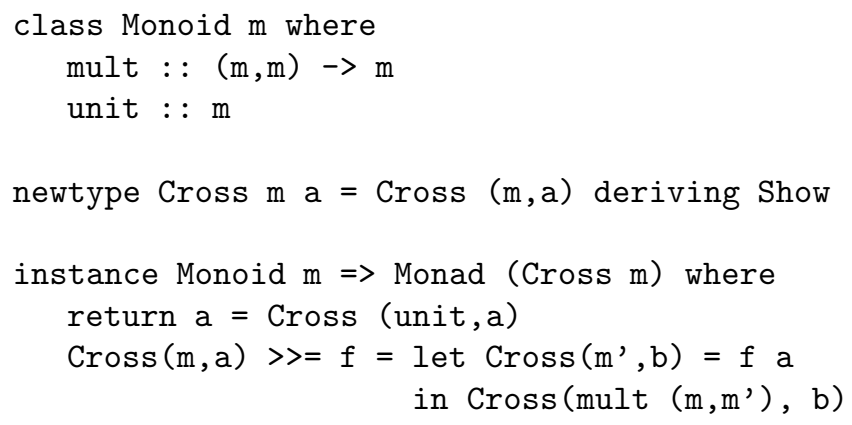

If we then apply our construction of a premonoidal Conway operator from the trace defined in Proposition 4.1 then we end up with an mfix operation of the type described by Launchbury and Erkök:

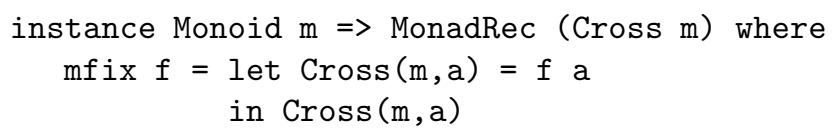

And this does have the expected behaviour:

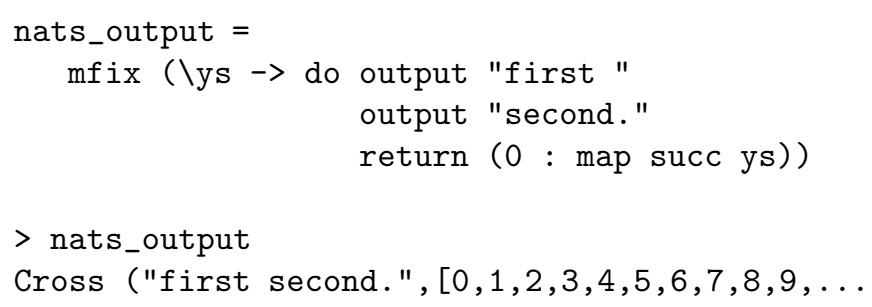

The two side effects have happened once only and in the order specified. 


\subsection{State}

Let $\mathbb{M}$ be a traced symmetric monoidal category, $S$ be an object of $\mathbb{M}$ and $\mathbb{K}$ be the category with the same objects as $\mathbb{M}$ and $\mathbb{K}(A, B)=\mathbb{M}(S \otimes A, S \otimes B)$ with the evident composition. If $\mathbb{M}$ is closed then $\mathbb{K}$ is equivalent to the Kleisli category of the state monad $T A=S \multimap S \otimes A$. Then $J: \mathbb{M} \rightarrow \mathbb{K}$ is premonoidal.

Proposition 4.2. In the above situation, the operation

$$
\hat{\operatorname{tr}}_{A, B}^{U}: \mathbb{K}(A \otimes U, B \otimes U) \rightarrow \mathbb{K}(A, B)
$$

defined by $\hat{\operatorname{tr}}_{A, B}^{U}(f)=\operatorname{tr}_{S \otimes A, S \otimes B}^{U}(f)$ is a premonoidal trace.

Again, the derived fixed point operator on the Kleisli category is easily defined in Haskell:

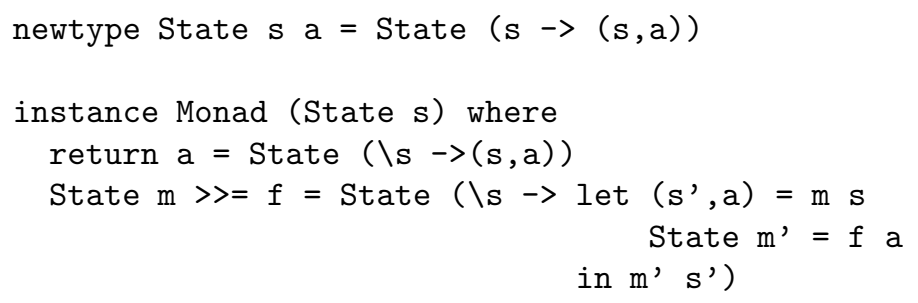

Note how the final value, a is recursively defined, but the final state $\mathbf{s}^{\prime}$ ' is not operationally, each time we go around the loop, the initial state is "snapped back" to $\mathrm{s}$.

\section{RELATED WORK}

Compared with Launchbury and Erkök's work on axiomatizing mfix, our definitions and results are in a rather more general setting, but account for rather fewer concrete examples. The axioms in [22] are almost identical to our definition of a premonoidal Conway operator except that they weaken some of our equations to inequations (interpreted in a concrete category of domains), add a strictness condition on one and regard some as additional properties which may hold in some cases (i.e. not part of the basic definition of what they call a "recursive monad"). These weaker conditions admit definitions of $\mathrm{mfix}$ for monads such as Maybe $(1+(\cdot))$, lazy lists and Haskell's IO monad [10] which do not have Conway operators satisfying our conditions.

Paterson has designed a convenient syntax for programming with Hughes's arrows (just as Haskell adds do to simplify programming with monads) [28, 29]. 
Paterson gives axioms for an ArrowLoop operation which are the same as our definition of a premonoidal trace; our results thus prove an equivalence between ArrowLoop and a particular (newly identified) class of mfixs.

Jeffrey [20] has also considered a variant of traces in a premonoidal setting, though his construction is rather different from ours: he considers a partial trace (only applicable to certain maps) on the category of values rather than on that of computations.

Friedman and Sabry have also investigated value recursion [13], though their approach is rather different from the axiomatic one which we and the others cited here have taken. They view the ability to define computations recursively as an additional effect and give a "monad transformer" which adds a state-based updating implementation of recursion to an arbitrary monad. Lifting the operations of the underlying monad to the new one is left to the programmer (and can generally be done in different ways). Moggi and Sabry have further developed this operational approach [26], giving a semantics for $\mathrm{mfix}$ in which the side-effecting body is first evaluated (without using the bound variable) and the resulting value is closed up by wrapping it in an ordinary (value) fix.

\section{Conclusions And Further WORK}

We have formulated and proved a natural generalization of the theorem relating traces and Conway operators to the case of premonoidal categories. This has applications to the semantics of some non-standard recursion and feedback operations on computations which have been found useful in functional programming.

It would be interesting to see if one could explain Launchbury and Erkök's weaker axiomatization in a more general setting. The natural thing to try here is to be more explicit about the presence of an abstract lifting monad, along the lines of [14]. This may also help establish a connection with the partial trace operation used by Jeffrey [20]. We would also like to have some more examples.

We are in the process of investigating the premonoidal version of the "geometry of interaction" construction, which traditionally embeds a traced monoidal category into a compact closed one. This is interesting from a mathematical view and may also have some connection to the building of layered protocol stacks from stateful components.

\section{A. Why "CONWAY" operators?}

We give a brief explanation in category-theoretic terms of the link between Conway operators and the work of John Horton Conway. Consider a category $\mathbb{C}$ with biproducts. The notions of biproduct and of traced symmetric monoidal category are self-dual. It follows therefore from Theorem 2.9 that to give a trace on such a $\mathbb{C}$ is equally to give a Conway operator in our sense and to give one in the dual sense favoured by Bloom and Ésik. Now restrict to the case where $\mathbb{C}$ is a Lawvere theory 
(or the opposite of one as in [4], but it amounts to the same thing). Let the generating object be $U$. A category with biproducts is enriched in commutative monoids (so we can add maps) and it follows that $\operatorname{End}_{\mathbb{C}}(U)=\mathbb{C}(U, U)$ is a rig (ring without negatives) in the terminology promoted by Lawvere and Schanuel. (Bloom and Ésik use the older "semi-ring".) $\mathbb{C}$ is completely determined by $\operatorname{End}_{\mathbb{C}}(U)$ : maps from $U^{n}$ to $U^{m}$ are given by $m \times n$ matrices over $\operatorname{End}_{\mathbb{C}}(U)$ (using the usual column vector conventions).

We consider a Conway operator on such a $\mathbb{C}$, that is, a matrix Conway theory for Bloom and Ésik [4]. They show the following

Theorem A.1. To give a Conway operator on a Lawvere theory $\mathbb{C}$ with biproducts is to equip the rig $\operatorname{End}_{\mathbb{C}}(U)$ with a unary operator $a \mapsto a^{*}$ satisfying the Conway identities

$$
\begin{aligned}
(a b)^{*} & =1+a(b a)^{*} b \\
(a+b)^{*} & =\left(a^{*} b\right)^{*} a^{*} .
\end{aligned}
$$

The Conway identities appear in [7] in the course of an analysis of the theory of regular languages.

Acknowledgements. We thank the referees and Zoltán Ésik for their helpful feedback.

\section{REFERENCES}

[1] R.S. Bird, Using circular programs to eliminate multiple traversals of data. Acta Informatica 21 (1984) 239-250.

[2] P. Bjesse, K. Claessen, M. Sheeran and S. Singh, Lava: Hardware design in Haskell, in International Conference on Functional Programming (1998).

[3] S.L. Bloom and Z. Ésik, Axiomatizing schemes and their behaviors. J. Comput. Syst. Sci. 31 (1985) 375-393.

[4] S.L. Bloom and Z. Ésik, Iteration Theories. EATCS Monographs on Theoretical Computer Science, Springer-Verlag (1993).

[5] V.E. Cazanescu and Gh. Stefanescu, A general result on abstract flowchart schemes with applications to the study of accessibility, reduction and minimization. Theor. Comput. Sci. 99 (1992) $1-63$.

[6] K. Claessen and D. Sands, Observable sharing for functional circuit description, in Asian Computing Science Conference (1999).

[7] J.H. Conway, Regular Algebra and Finite Machines. Chapman Hall (1971).

[8] R.L. Crole and A.M. Pitts, New foundations for fixpoint computations: FIX-hyperdoctrines and the FIX-logic. Inf. Comput. 98 (1992) 171-210.

[9] L. Erkök, Value Recursion in Monadic Computations. Ph.D. thesis, Oregon Graduate Institute, OHSU (October 2002)

[10] L. Erkök, J. Launchbury and A. Moran, Semantics of value recursion for monadic input/output. RAIRO Theoret. Informatics Appl. 36 (2002) 155-180.

[11] Z. Ésik, Axiomatizing iteration categories. Acta Cybernet. 14 (1999).

[12] Z. Ésik, Group axioms for iteration. Inf. Comput. 148 (1999) 131-180

[13] D.P. Friedman and A. Sabry, Recursion is a computational effect. Technical Report 546, Computer Science Department, Indiana University (December 2000). 
[14] C. Fuhrmann, A. Bucalo and A. Simpson, An equational notion of lifting monad. Theor. Comput. Sci. 294 (2003) 31-60.

[15] J.-Y. Girard, Towards a geometry of interaction, in Categories in Computer Science and Logic, edited by J.W. Gray and A. Scedrov. Contemp. Math. 92 (1989) 69-108.

[16] M. Hasegawa, Models of Sharing Graphs (A Categorical Semantics of Let and Letrec). Distinguished Dissertations in Computer Science, Springer-Verlag (1999).

[17] M. Hasegawa, The uniformity principle on traced monoidal categories, edited by R. Blute and P. Selinger. Elsevier, Electronic Notes in Theor. Comput. Sci. (2003).

[18] J. Hughes, Generalising monads to arrows. Sci. Comput. Program. 37 (2000) 67-112.

[19] M. Hyland and A.J. Power, Symmetric monoidal sketches, in Proc. of the 2nd Conference on Principles and Practice of declarative Programming (2000) 280-288.

[20] A. Jeffrey, Premondoidal categories and a graphical view of programs. http://www.cogs . susx.ac.uk/users/alanje/premon/ (June 1998).

[21] A. Joyal, R. Street and D. Verity, Traced monoidal categories. Math. Proc. Cambridge Philoso. Soc. 119 (1996).

[22] J. Launchbury and L. Erkök, Recursive monadic bindings, in International Conference on Functional Programming (2000).

[23] J. Launchbury, J.R. Lewis and B. Cook, On embedding a microarchitectural design language within Haskell. International Conference on Functional Programming (1999).

[24] J. Launchbury and S.L. Peyton Jones, State in Haskell. Lisp and Symbolic Computation 8 (1995) 293-341.

[25] E. Moggi, Notions of computation and monads. Inf. Comput. 93 (1991) 55-92.

[26] E. Moggi and A. Sabry, An abstract monadic semantics for value recursion, in Proc. of the 2003 Workshop on Fixed Points in Computer Science (April 2003).

[27] P.S. Mulry, Strong monads, algebras and fixed points, in Applications of Categories in Computer Science, edited by M.P. Fourman, P.T. Johnstone and A.M. Pitts. LMS Lecture Notes 177 (1992) 202-216.

[28] R. Paterson, A new notation for arrows, in Proc. of the International Conference on Functional Programming. ACM Press (September 2001).

[29] R. Paterson, Arrows and computation, in The Fun of Programming, edited by J. Gibbons and O. de Moor. Palgrave (2003) 201-222.

[30] A.J. Power and E.P. Robinson, Premonoidal categories and notions of computation. Math. Struct. Comput. Sci. 7 (1997) 453-468.

[31] A.J. Power and H. Thielecke, Closed Freyd and $\kappa$-categories. International Conference on Automata, Languages and Programming (1999).

[32] A.K. Simpson and G.D. Plotkin, Complete axioms for categorical fixed-point operators, in Proc. of 15th Annual Symposium on Logic in Computer Science. IEEE Computer Society (2000).

[33] P. Wadler, The essence of functional programming, in Proc. of the 19th Symposium on Principles of Programming Languages. ACM (1992).

Received November 12, 2002. Accepted in final form August 14, 2003. 